\title{
Recuperación del atractivo migratorio metropolitano en el período 2004-2009: ¿factores exógenos o endógenos? ${ }^{1}$
}

\author{
Jorge Rodríguez Vignoli² y Daniel Espinoza ${ }^{3}$
}

\begin{abstract}
RESUMEN
Se examinan dos asuntos relacionados con las tendencias de la localización y la movilidad espacial de la población chilena. El primero atañe a la pérdida de atractivo migratorio registrada por la Región Metropolitana y el Área Metropolitana del Gran Santiago en los últimos años, que podría moderarse o revertirse como resultado de procesos estructurales que fortalecen el papel de las metrópolis en el desarrollo mundial y nacional y de una coyuntura adversa para economías de regiones que se habían convertido en alternativa migratoria para los habitantes de Santiago. El segundo asunto atañe a la bien documentada expansión periférica, a los signos de suburbanización del estrato alto y al conocido despoblamiento del centro, que podrían tender a moderarse por una combinación de factores entre los que se encuentran los crecientes costos monetarios y de tiempo de los desplazamientos, las renovadas opciones habitacionales ofrecidas por el Cono Oriente y las políticas públicas destinadas a promover la residencia en el centro y pericentro. Las tendencias del atractivo migratorio regional, metropolitano y comunal se miden usando microdatos censales y de encuestas CASEN, que desde 2006 han incorporado un módulo de migración. Los principales hallazgos del análisis empírico, que debe ser considerado como preliminar y exploratorio, centrado en las novedades que ofrece la CASEN 2009 para el período 20042009, tiende a respaldar, con matices en algunos casos, estas hipótesis, aunque se necesitan estudios más detallados para conclusiones más sólidas al respecto.
\end{abstract}

Palabras clave: Migración interna interregional, suburbanización, expansión periférica, renovación urbana.

\begin{abstract}
Two issues related to the trends of localization and spatial mobility of the Chilean population, are examined. The first concerns the loss in recent years of the migratory attractiveness registered in the Metropolitan Region and in the Metropolitan Area of Greater Santiago, which could ease or reverse as a result of structural processes that strengthen the role of the metropolis in global and national development, and also as a consequence of adverse circumstances facing the economies of regions which had lately become a migratory alternative for Santiago's inhabitants. The second issue concerns the well-documented process of peripheral expansion, the "suburbanization" of the upper social stratum and the depopulation of downtown areas, which could tend to abate due to a number of factors, among which include increasing costs and commuting times, renewed housing options offered in the "East End" and public policies aimed at promoting living downtown and surrounding areas. The trends of regional, metropolitan and communal migratory attractiveness are measured using census micro data and the CASEN survey, which has incorporated a migration module since 2006. The main findings of the empirical analysis, which is to be considered preliminary and exploratory, focused on the new evidence offered by the CASEN 2009 survey for the 2004-2009 period, tend to support, with nuances in some cases, these hypotheses, although further detailed studies would be required to establish more solid conclusions.
\end{abstract}

Key words: Internal migration, suburbanization, urban sprawl, urban renewal.

\footnotetext{
1 Artículo recibido el 4 de enero de 2011, aceptado el 7 de dciembre de 2011 y corregido el 22 de diciembre de 2011.

2 CELADE-División de Población de la Comisión Económica para América Latina y el Caribe (CEPAL) de las Naciones Unidas (Chile). E-mail: jorge.rodriguez@cepal.org
}

\footnotetext{
3 Sociólogo, Universidad de Chile (Chile). E- Mail: daniel.espinoza.carrasco@gmail.com
} 
Chile, como varios otros países de América Latina, se ha caracterizado por una alta concentración demográfica, económica y política en la ciudad más poblada, en este caso Santiago que es a la vez la capital y que se ubica en la Región Metropolitana, una de las quince regiones en que se divide actualmente el país. Esta alta concentración ha sido objeto de críticas diversas ${ }^{4}$ por lo cual desde la década de 1960 diferentes gobiernos han procurado, al menos formalmente, reducirla mediante una gama de políticas de desconcentración territorial, tales como programas de colonización, de regionalización y de desarrollo regional, de estímulos e incentivos para empresas y personas vinculadas a localización fuera de la ciudad más poblada, de traslado de instalaciones de poderes del Estado y de servicios públicos fuera de la ciudad más poblada, de descentralización político administrativa, etc. (Rodríguez y Busso, 2009). Desde mediados de la década de 1970 las intervenciones comenzaron a cambiar producto del modelo político y económico imperante. Este modelo se basa en la apertura comercial, la desregulación y el rol central del mercado. A causa de los dos últimos atributos, se comenzó a desactivar la acción estatal directa. Y con base en el primer rasgo se anticipó un dinamismo reciente de los rubros económicos con ventajas comparativas en el concierto mundial. Dado que estos rubros son básicamente primarios y se localizan en regiones diferentes a la Región Metropolitana, la expectativa era que este mayor dinamismo iba a generar un contrapeso estratégico a la histórica concentración económica y demográfica en Santiago (Daher, 1994).

\footnotetext{
4 En un documento reciente, el BID plantea que “...the 'excess of urbanization' (in Latin America) is in large part due to the disproportionate growth of the region's large metropolises..." (BID, 2011: 6). Las críticas a esta concentración tienen un componente económico. Basándose en la hipótesis de Williamson y su reformulación por Henderson, se plantea que pasado cierto umbral de desarrollo económico la concentración deja de ser funcional y que, en promedio, la concentración excesiva implica pérdidas substanciales de ingreso (ILPES, 2009: 30). Y también tienen un componente sociopolítico. Se plantea que acumulan problemas y déficits justamente por su gran tamaño y las dificultades para gobernarlas.
}

Numerosos autores han cuestionado estas expectativas, varios de ellos rescatando las ventajas y las bondades de la concentración y de las áreas metropolitanas. ${ }^{5}$ Otros autores, críticos de la concentración, también cuestionan estas expectativas a causa de la existencia de fuerzas globales y locales que contrarrestan los potenciales efectos "desconcentradores" del modelo económico. En esa línea, Aroca (2009) identifica tanto mecanismos públicos -el centralismo del presupuesto nacional, la asignación de becas de Estado para capital humano avanzado y la política monetaria del Banco Central de Chile para controlar la inflación- como de mercado -creciente "conmutación interregional", comercio interregional, alta concentración de la gerencias o entidades de decisión del sector privado en Santiago- entre los responsables de la persistente disparidad regional y la concentración en torno a la Región Metropolitana.

La evidencia disponible, por su parte, puede considerarse ambigua e inconcluyente aún en el plano de la concentración económica. El trabajo de Aroca (2009) sirve como referencia al respecto. Este autor encuentra en su análisis de las series de producción regional entre 1992 y 2003 que, efectivamente el crecimiento de la producción de las regiones ha sido más alto que el de la Región Metropolitana, pero que los ingresos han aumentado más en esta última. Desde la perspectiva demográfica, la Región Metropolitana aumentó su peso relativo dentro de la población nacional entre 1992 y 2002, pero por vez primera experimenta un saldo migratorio negativo entre 1997 y 2002 (Rodríguez, 2008).

\footnotetext{
5 El título de un artículo de Carlos de Mattos (1999) es elocuente: "Santiago de Chile, globalización y expansión metropolitana: lo que existía sigue existiendo". En esta revalorización de las metrópolis, los planteamientos sobre las ciudades globales y las regiones urbanas (Sassen, 1991; 2007) fueron claves. Y en la reivindicación de la concentración tanto la denominada "nueva geografía económica" (Fujita et al., 2000) como documentos de posición de organismos multilaterales, ha sido cruciales: "Cuando funcionan bien, los mercados de tierras, mano de obra y productos consiguen la eficiencia económica que acompaña a la concentración geográfica, y la equidad asociada con la convergencia de los niveles de vida" (Banco Mundial, 2008: 22).
} 
Ahora bien desde 2006 la Encuesta de Caracterización Socioeconómica Nacional (CASEN) incluye un módulo de migración que permite estimar el atractivo migratorio a escala regional y comunal (esto último solo en algunos casos). La encuesta CASEN 2006 ya fue usada con tales propósitos (Rodríguez, 2008) y sus resultados ratificaron los del censo en torno a la emigración neta de la Región Metropolitana, lo que abonó a las hipótesis "desconcentradoras".

En tal sentido, el objetivo del presente estudio con relación al tema de la concentración demográfica en Santiago es aprovechar la disponibilidad de la encuesta CASEN 2009 para continuar con el monitoreo de las tendencias de la migración interregional, en tanto indicador clave de la eventual de desconcentración regional, y evaluar, en particular, el impacto de una crisis económica regional, la de la industria del salmón, sobre el atractivo migratorio que mostró la Región de Los Lagos en el censo de 2002 y la CASEN de 2006.

Asimismo, pero por razones diferentes, se examina el atractivo migratorio de la Región Metropolitana de Santiago (RMS) y del Ârea Metropolitana del Gran Santiago (AMGS: 32 comunas de la provincia de Santiago más las comunas de Puente Alto y San Bernardo). Por un simple efecto compensador de las crisis económicas regionales, la RMS y el AMGS deberían experimentar un repunte de su atractivo migratorio. Lo anterior podría ser, incluso favorecido por el dinamismo de las economías regionales basadas en la exportación de minerales, en particular cobre, merced a la capacidad de apropiación del ingreso y la conmutación interregional descritos por Aroca para la RM. Y ciertamente la evolución socioeconómica y ambiental de la RM y del AMGS podría contribuir a la recuperación de su atractivo, en caso de ser una evolución favorable.

\section{Sobre la reconfiguración del Área Metropolitana del Gran Santiago: ¿son la expansión periférica y el despoblamiento del centro inevitables?}

La investigación urbana mundial, fuertemente influenciada por la experiencia de los países actualmente desarrollados y llevada a cabo preferentemente por investigadores $y$ entidades de esos países, ha ido construyendo un relato sobre los patrones de uso del espacio y la configuración territorial y social de las grandes ciudades. Con frecuencia, esta descripción estilizada se presenta con una cierta fuerza normativa, sea porque tiene fundamentos teóricos sólidos ${ }^{6}$ o sea por el solo hecho de que se le presenta como "regularidades que aparecen en casi todas las grandes ciudades del mundo" (Galetovic y Jordán, 2006: 125). Cualquiera sea el caso, este relato tiende a considerar como uno de sus hitos a la reducción de la densidad media, lo que sería resultado de la expansión periférica, la suburbanización y el paulatino despoblamiento de la zona céntrica, siendo la fuerza subyacente de lo anterior el aumento de los ingresos (Galetovic y Jordán, 2006: 125).

EI AMGS ha seguido de una manera heterodoxa esta evolución. Experimentó despoblamiento del centro y expansión periférica, pero, en línea con lo observado en casi todas las grandes ciudades de América Latina (Torres, 2008), esta última se dio por la ocupación de grupos pobres, con lo cual en algunas zonas de la periferia se alcanzaron las densidades más altas de la ciudad, lo que no está previsto por la el relato hegemónico (Galetovic y Jordán, 2006: 125-126).

\footnotetext{
6 "In general, the classical theory predicts that variations in urban land cover, average density, and fragmentation among cities and countries, as well as their rates of change, can be largely explained by variations in city population, household income, buildable land, the cost of agricultural land in the urban periphery, and the cost of urban transport... The evidence presented in this report validates the key results of their theoretical insights and confirms the observation of Mills and Tan $(1980,314)$ that [t]here are few cases in economics in which such a simple theory leads to so many testable implications" (Angel et al., 2011: 5).
} 
Por otro lado, en línea con la experiencia de las ciudades de países desarrollados, desde mediados de la década de 1990 son visibles los procesos de suburbanización de grupos de ingreso medio y alto, básicamente bajo la modalidad de barrios cerrados o parcelaciones relativamente aisladas (Sabatini y Cáceres, 2004). Sin embargo, a contrapelo del relato hegemónico, desde mediados de la década de 1990 es visible el empuje inmobiliario en el centro y en ciertas zonas del pericentro, en virtud de políticas públicas (subsidio de renovación urbana); aunque la recuperación demográfica parece estar rezagada respecto del dinamismo inmobiliario, al menos para la comuna de Santiago (Rodríguez, 2007; 2008), se espera que finalmente también se verifique una recuperación del crecimiento de la población por la llegada de inmigrantes. Respecto de esto último, un asunto no abordado por la literatura y que se considerará en este trabajo, atañe al origen de estos inmigrantes (¿Intrametropolitanos?, ¿de otras regiones?, ¿de otros países?).

Así, durante el período cubierto por la CASEN 2009 para medir la migración (20052009), se verificaron al menos dos fenómenos claves para la reconfiguración socioespacial del AMGS. Por una parte, por vez primera en varias décadas se enfrentan las fuerzas que promueven la expansión periférica (menor costo del suelo, búsqueda de ganancia de agentes inmobiliarios, políticas públicas de construcción de vivienda social, menor control urbano, normativas urbanas menos estrictas, expansión del metro, construcción de autopistas urbanas, etc.) con aquellas que favorecen la localización céntrica (en particular, subsidio de renovación urbana). La migración que experimenta la metrópolis es clave para dirimir si estas últimas están teniendo algún efecto. En segundo lugar, la suburbanización incipiente del estrato alto (Rodríguez, 2008; Sabatini y Cáceres, 2004) enfrenta desafíos emergentes dados por la maduración de los proyectos iniciales -reveladora de sus fortalezas y debilidades-, y por la capacidad de reinvención del cono oriente, sea mediante ampliación hacia faldeos precordilleranos o la densificación, en particular mediante construcción en altura. Por ello, un segundo objetivo de este trabajo es indagar empíricamente en ambos fenómenos, con especial referencia a la migración intrametropolitana, usando la encuesta CASEN 2009 como fuente de datos.

El documento se compone de cinco secciones adicionales a esta introducción. En la segunda, se presenta de manera muy escueta el marco metodológico, el que describe la fuente de datos, los indicadores y los principales procesamientos que se usarán en el estudio. Dado que se trata de un trabajo básicamente descriptivo, su componente metodológico es simple. En la tercera sección se examina la evolución de la migración interregional, con énfasis en las novedades que ofrece la CASEN 2009 para el período 20052009 y con el foco del análisis en algunas regiones seleccionadas, entre ellas las de Los Lagos y la Metropolitana. En la cuarta sección se cuantifica y describe el intercambio migratorio del Área Metropolitana del Gran Santiago con el fin de verificar si se mantiene la emigración neta o hay indicios de repunte del atractivo migratorio. La quinta sección examina el impacto de la migración, incluyendo la intercomunal intrametropolitana, sobre la expansión y la reconfiguración socioterritorial del Área Metropolitana del Gran Santiago. La sexta ofrece las principales conclusiones y desafíos de investigación que se desprenden del estudio.

\section{Marco metodológico}

Se usarán los microdatos ${ }^{7}$ de los censos de 1992 y 2002 y de las encuestas CASEN 2006 y 2009 para estimar tasas de migración interna neta por regiones y comunas. Para abordar la primera cuestión del documento, relativa al atractivo migratorio de las regiones en el marco de la evolución económica y social nacional y regional durante el período 2004-2009, se construirán las matrices de migración interna interregional y se calcularán con ellas las tasas de migración neta media anual del período ${ }^{8}$. El principal factor asocia-

\footnotetext{
7 Bases descargables de manera gratuita, previo llenado de formulario de solicitud. Disponible en Internet: http://www.ministeriodesarrollosocial.gob. cl/casen/bases_datos.html

8 Se usaron la comuna de empadronamiento (coincide con la de residencia habitual por ser la CASEN una encuesta de derecho) y la consulta "En qué comuna o lugar vivía usted en noviembre de 2004".
} 
do a la tendencias del atractivo migratorio de las regiones será la tasa de desempleo abierta captada por la misma encuesta CASEN. Por el carácter esencialmente descriptivo del trabajo, no se modelará ni la tendencia ni el diferencial en 2009 en materia de atractivo migratorio sino que se pondrá atención específica a los dos casos de interés: la Región Metropolitana y la Región de Los Lagos.

Respecto de la segunda cuestión del estudio, el efecto de la migración sobre la expansión y la reconfiguración socioterritorial del Área Metropolitana del Gran Santiago, se usarán tres matrices de migración para calcular flujos, saldos y tasas migratorias. La primera tendrá solo tres entidades -AMGS, resto de la Región Metropolitana y resto del país-y con ella podrá evaluarse tanto el intercambio migratorio del AMGS cuanto su componente "cercano", eventualmente asociado a suburbanización o a ampliación metropolitana difusa, como en el "lejano, más propio de la desconcentración, aunque también puede obedecer a procesos de conformación de "regiones urbanas" en la terminología de Sassen (2007). La segunda matriz captará solo el intercambio migratorio intrametropolitano, pero lo hará para las 34 comunas del AMGS. Por su extensión no será expuesta en el documento, pero sus resultados se reflejarán en mapas que identificarán a las comunas según su condición de saldo migratorio positivo o negativo. $Y$ la tercera matriz, utiliza agrupaciones de comunas del AMGS -zonas sociogeográficas de la ciudad, que serán identificadas y explicadas debidamente- y las agrupaciones de comunas del resto de la RM y de fuera de la RM para captar el atractivo migratorio de estas zonas en su intercambio con el resto de la ciudad y el resto del país.

Por tanto, la medición es válida para el período noviembre 2004 a noviembre 2009. Para detalles sobre el cálculo de la tasa ver Rodríguez y Busso (2009).

\section{La migración interregional en el período 2004-2009: inflexiones migratorias varias, agudización de la expulsión y el puzle de la Región de Antofagasta}

La CASEN 2009 muestra cambios significativos del patrón migratorio interregional ya que de un cuadro relativamente equilibrado de regiones atractivas y expulsoras captado por el censo de 2002 y la CASEN de 2006 se pasa a uno muy polarizado con solo dos regiones atractivas y el resto expulsoras. En tal sentido, el principal hallazgo de la CASEN 2006 es que tras una década de saldos negativos, la Región Metropolitana recupera su atractivo migratorio en el período 2004-2009 (Cuadro No 1). Y la magnitud de su saldo migratorio es notable, pues la ganancia de efectivos de los últimos 5 años más que duplica la pérdida acumulada en los últimos 10 años. El mismo cuadro muestra una reducción significativa del atractivo de la Región de Los Lagos, un aumento de la emigración de las regiones históricamente expulsoras (del Maule, del Biobío y de la Araucanía) y una inflexión del atractivo de la Región de Valparaíso.

Las potenciales explicaciones para esta recuperación del atractivo no pueden, en principio, basarse en la superioridad de la RM en varios indicadores socioeconómicos clave -ingresos medios mayores, mayor cobertura educativa, mayor acceso a servicios básicos y nuevas tecnologías-, porque tal superioridad también existía en los períodos previos en que la RM registró emigración neta (Fuentes, 2011). Tampoco puede imputarse al mercado de trabajo de la RM, porque tal como se aprecia en el Cuadro $N^{\circ}$ 2 la tasa de desempleo de la RM tuvo un aumento incluso superior al observado a escala nacional entre 2006 y 2009. Con todo, esta recuperación del atractivo de Santiago sí podría deberse, al menos en parte, a un efecto acumulado de avances en ciertos problemas clave de la ciudad, como la contaminación, la infraestructura vial, el déficit habitacional y la pobreza (Fuentes, 2011; Galetovic y Jordán, 2006) y a otros factores de mayor alcance como las creciente facilidades para 
conmutar a regiones, en línea con lo planteado por Aroca (2009). Más indagación específica sobre estos factores y su evolución comparativa con el resto de las regiones será necesario para evaluar esta posibilidad.

Respecto de la significativa merma del atractivo de la Región de Los Lagos, parte importante del período de referencia estuvo marcado por la crisis de la industria del salmón, motor económico y laboral de la X Región de Puerto Montt; de hecho, tanto el censo de 2002 como la CASEN de 2006 habían mostrado que esta región se había convertido en centro de atracción de migrantes de todo el país (Rodríguez, 2008). El Cuadro $\mathrm{N}^{\circ} 2$ muestra el efecto más relevante de esta crisis para la migración, a saber el abrupto aumento de la tasa de desempleo en dicha región que se duplica entre 2006 y 2009, vale decir sufre un aumento mucho mayor que el promedio nacional al punto de superar la media nacional en 2009.

También hubo situaciones difíciles, ninguna tan grave como la del salmón eso sí, en otros rubros exportadores de productos primarios (ciclo de bajos precios de la celulosa y de bajas capturas pesqueras, por ejemplo). Como estas actividades productivas se localizan principalmente en regiones distintas a la metropolitana, el efecto de sus decaimientos puede haber ido en desmedro de la capacidad de atracción de las regiones donde se localizan. De hecho, el significativo aumento de la desocupación en la Región de La Araucanía (Cuadro $N^{\circ}$ 2) puede haber contribuido a la significativa alza de su emigración.

Por último, cabe destacar el caso de la Región de Antofagasta, cuyo desempleo en el período de referencia está bien por debajo de la media nacional (Cuadro $N^{\circ} 2$ ) y a diferencia del promedio nacional no aumentó en el período 2006-2009; además, su ingreso medio está bien por encima de la media nacional (Ministerio de Desarrollo Social, 2009). Y pese a todos estos factores que determinan atractivo relativo de esta región para los migrantes, registra según la CASEN 2009 (y también la de 2006) un saldo negativo. Sin pretender explicar esta paradoja, cabe mencionar, en línea con lo planteado por Aroca (2009), que esta región es, por lejos, la que registra mayor conmutación laboral. Y la encuesta CASEN 2009 ratifica esta condición, pues capta una cifra del orden de 26.500 trabajadores en ella ${ }^{9}$ pero que residen en otras regiones del país, lo que significa el $11 \%$ de los ocupados de la región. En tal sentido, al menos para esta región, la conmutación podría ser una alternativa a la migración laboral y ello podría explicar, al menos en parte, el saldo migratorio negativo de la región.

En síntesis, la recuperación del atractivo de la RM parece deberse tanto a ciertas fortalezas internas, o si se quiere una reducción de ciertas debilidades internas, como a una coyuntura particularmente complicada para varias regiones, algunas de ellas, sobre todo la de Los Lagos, alternativas migratorias para la población de la RM en años anteriores. Por ello habrá que esperar a una recuperación del escenario regional para evaluar con mayor rigor la sostenibilidad de esta recuperación del atractivo de la RM.

9 Procesamientos de los autores, cuyos resultados no se muestran en el documento, pero están disponibles a solicitud. 
Cuadro $\mathrm{N}^{\circ} 1$

Chile: Migración reciente interregional (saldo y tasa), censo 2002 (1997-2002), CASEN 2006 (2002-2006) y CASEN 2009 (2004-2009), población de 5 años y más.

\begin{tabular}{|c|c|c|c|c|c|c|}
\hline \multirow[b]{2}{*}{ Región } & \multicolumn{3}{|c|}{ Migración neta } & \multicolumn{3}{|c|}{ Tasa Migración neta (por mil) } \\
\hline & $\begin{array}{l}1997 \\
2002\end{array}$ & $\begin{array}{l}2002 \\
2006\end{array}$ & $\begin{array}{l}2004 \\
2009\end{array}$ & $\begin{array}{l}1997 \\
2002\end{array}$ & $\begin{array}{l}2002 \\
2006\end{array}$ & $\begin{array}{l}2004 \\
2009\end{array}$ \\
\hline Tarapacá & 5.063 & 3.254 & 14.303 & 5.0 & 3.0 & 10.6 \\
\hline Antofagasta & 2.701 & -2.043 & -10.611 & 1.3 & -0.9 & -4.1 \\
\hline Atacama & -5.919 & -1.632 & -102 & -5.2 & -1.5 & -0.1 \\
\hline Coquimbo & 12.261 & 783 & 100 & 4.6 & 0.3 & 0.0 \\
\hline Valparaíso & 21.211 & 4.592 & -13.782 & 3.1 & 0.7 & -1.7 \\
\hline Gral. B. O'Higgins & 4.382 & 8.059 & -10.384 & 1.2 & 2.2 & -2.5 \\
\hline Maule & -1.712 & -1.344 & -12.075 & -0.4 & -0.3 & -2.6 \\
\hline Biobío & -18.764 & -7.956 & -29.780 & -2.2 & -1.0 & -3.1 \\
\hline La Araucanía & -1.854 & -2.698 & -19.896 & -0.5 & -0.7 & -4.4 \\
\hline Los Lagos & 10.588 & 13.143 & 2.752 & 3.4 & 4.0 & 0.7 \\
\hline Aysén & -235 & -1.266 & -5.340 & -0.6 & -3.3 & -10.9 \\
\hline Magallanes y Antártica Chilena & -4.534 & 6.197 & 189 & -6.7 & 10.3 & 0.3 \\
\hline Metropolitana & -12.229 & -16.992 & 98.704 & -0.5 & -0.6 & 3.2 \\
\hline Los Ríos & -6.977 & -4.514 & -7.137 & -4.3 & -2.9 & -4.0 \\
\hline Arica y Parinacota & -3.982 & 2.417 & -6.941 & -4.7 & 3.2 & -8.2 \\
\hline
\end{tabular}

Fuente: Elaboración propia en base a procesamiento especial de microdatos del censo de 2002 y de las encuestas CASEN 2006 y 2009.

Nota: resultados obtenidos usando factor de expansión regional. 
Cuadro $\mathrm{N}^{\circ} 2$

Chile: Tasa de desempleo regional, 1990-2009

\begin{tabular}{|l|r|r|r|r|r|r|r|r|r|}
\hline & \multicolumn{7}{|c|}{ Tasa de desempleo } \\
\cline { 2 - 10 } Región & 1990 & 1992 & 1994 & 1996 & 1998 & 2000 & 2003 & 2006 & 2009 \\
\hline Arica y Parinacota & - & - & - & - & - & - & - & 8,6 & 9,3 \\
Tarapacá & 8,5 & 3,8 & 8,0 & 5,6 & 8,5 & 11,3 & 10,4 & 9,7 & 6,1 \\
Antofagasta & 7,5 & 7,4 & 6,1 & 4,5 & 6,6 & 11,4 & 8,5 & 6,1 & 5,8 \\
Atacama & 6,9 & 4,9 & 4,0 & 5,5 & 11,3 & 11,2 & 10,1 & 6,5 & 9,4 \\
Coquimbo & 8,2 & 5,8 & 7,8 & 6,3 & 9,9 & 11,2 & 9,1 & 7,0 & 8,0 \\
Valparaíso & 10,4 & 5,7 & 7,5 & 6,8 & 10,3 & 11,8 & 12,1 & 8,3 & 12,0 \\
Gral. B. O'Higgins & 8,7 & 4,4 & 8,3 & 6,6 & 8,4 & 8,0 & 7,9 & 6,2 & 7,5 \\
Maule & 6,6 & 5,2 & 7,3 & 4,9 & 10,3 & 8,3 & 9,4 & 6,8 & 9,2 \\
Biobío & 10,1 & 8,7 & 8,0 & 7,3 & 13,4 & 12,3 & 11,0 & 9,9 & 12,6 \\
La Araucanía & 7,4 & 4,9 & 5,2 & 6,8 & 8,5 & 11,5 & 10,9 & 7,5 & 13,2 \\
Los Ríos & - & - & - & - & - & - & - & 8,2 & 11,3 \\
Los Lagos & 7,7 & 4,7 & 7,3 & 6,2 & 8,9 & 11,5 & 8,4 & 5,0 & 10,5 \\
Aysén & 7,9 & 5,3 & 8,3 & 4,6 & 4,9 & 5,3 & 7,7 & 2,9 & 6,9 \\
Magallanes y & 7,9 & 3,9 & 3,2 & 4,8 & 6,3 & 5,4 & 6,6 & 4,7 & 8,3 \\
Antártica Chilena & 7,9 & 5,2 & 5,9 & 4,9 & 9,6 & 9,8 & 9,3 & 7,0 & 10,1 \\
Metropolitana & 8,3 & 5,6 & 6,7 & 5,7 & 9,9 & 10,4 & 9,7 & 7,3 & 10,2 \\
Total país & 0,947 & 0,924 & 0,884 & 0,865 & 0,977 & 0,944 & 0,962 & 0,953 & 0,987 \\
Rel RM/total & 0,929 & 0,851 & 1,094 & 1,083 & 0,900 & 1,103 & 0,865 & 0,687 & 1,026 \\
Rel X/total & & & & & & & & \\
\hline
\end{tabular}

\section{El Área Metropolitana del Gran Santiago: el retorno del atractivo para los migrantes de otras regiones}

Al igual que en el caso de la Región Metropolitana, la CASEN 2009 indica que el Área Metropolitana del Gran Santiago (AMGS) quiebra la tendencia registrada desde mediados de la década de 1990, pues aparece como una ciudad atractiva para los migrantes, con una inmigración neta de 63.997 personas (Cuadro No 3). El resto de la Región Metropolitana (es decir, las comunas fuera del AMGS) también tienen un saldo positivo, aunque sus números absolutos sean netamente inferiores. Lo anterior no significa que "el resto" sea menos atractivo, ya que las bases numéricas de ambos territorios difieren significativamente (el AMGS representa el $90 \%$ de la población de la RM), de hecho, al considerar las tasas de migración neta (no mostradas en este documento), la intensidad de la inmigración neta es mayor en el caso del "resto de la RM". Más aún, el saldo del intercambio migratorio entre ambas zonas favorece al "resto" de la RM, en línea con lo registrado por el censo de 2002 y la CASEN de 2006 (Cuadro No 4). 
Estos resultados avalan la persistencia de un proceso de expansión extraperiférica (suburbanización) de la metrópolis de Santiago, que está bien documentado y que se dirige hacia distintas localidades del entorno de la ciudad, ubicadas entre 40 y 70 kilómetros del centro de la misma (De Mattos, 2001). Por lo mismo, y en línea con los argumentos desarrollados para la Región Metropolitana, el atractivo del Gran Santiago que detecta la CASEN 2009, probablemente esté determinado por el deterioro del atractivo relativo de ciudades o ámbitos alternativos de residencia en otras regiones del país ${ }^{10}$.

De hecho, esto es lo que se evidencia con el Cuadro $N^{\circ} 3$, sobre todo porque muestra una caída del orden del $70 \%$ en la emigración del AMGS hacia comunas fuera de la RM, mientras que la corriente inversa se mantiene en los volúmenes detectados por la

10 También puede deberse a la sustitución de la migración hacia ellas por otras modalidades de desplazamiento físicas o virtuales. Esa posibilidad será investigada en futuros estudios.
CASEN 2006. Se trata de una baja muy brusca y cuyos fundamentos de última instancia - ¿aumentó el atractivo del AMGS para los santiaguinos o se redujo el atractivo del resto del país para los santiaguinos?: ambas opciones llevan al mismo resultado: alza de la retención del AMGS- deberán ser objeto de investigación ulterior.

Respecto del proceso de suburbanización, el Cuadro $\mathrm{N}^{\circ} 4$ es elocuente en evidenciar su persistencia, aunque en este caso lo que se mantiene es el nivel del flujo de salida, mientras se reduce sensiblemente la cuantía del flujo de llegada, que solo alcanza a 12.827 persona entre 2004 y 2009. Este saldo migratorio negativo del AMGS con su entorno cercano (resto de la Región Metropolitana) podría validar ciertas teorías de la "ciudad difusa" (De Mattos, 2010; Ingram, 1997). Sin embargo, resultados relativos al atractivo migratorio del centro de la ciudad y del denominado cono oriente que se presentan más adelante en este mismo texto, cuestionan las visiones de una suburbanización sin contrapesos.

\section{Cuadro $\mathrm{N}^{\circ} 3$}

Migración entre AMGS, comunas fuera del AMGS y resto del país para el período 2004-2009, población de 5 años y más

\begin{tabular}{|l|r|r|r|r|r|}
\hline \multirow{2}{*}{$\begin{array}{l}\text { Residencia en noviembre de } \\
2009\end{array}$} & \multicolumn{2}{|c|}{ Residencia en noviembre de 2004 } & \multicolumn{2}{|c|}{$\begin{array}{c}\text { Total en } \\
\text { noviembre } \\
\text { de 2009 }\end{array}$} & $\begin{array}{c}\text { Migración } \\
\text { Neta }\end{array}$ \\
\cline { 1 - 4 } & AMGS & $\begin{array}{r}\text { Resto Región } \\
\text { Metropolitana }\end{array}$ & Resto País & & \\
\hline AMGS & 5.401 .143 & 12.827 & 119.284 & 5.533 .254 & 63.997 \\
\hline Resto Región Metropolitana & 29.731 & 706.760 & 10.285 & 746.776 & 17.709 \\
\hline Resto País & 38.383 & 9.480 & 9.333 .042 & 9.380 .905 & -81.706 \\
\hline Total en noviembre de 2004 & 5.469 .257 & 729.067 & 9.462 .611 & 15.660 .935 & - \\
\hline
\end{tabular}

Fuente: Procesamiento especial de microdatos de la encuesta CASEN 2009. 


\section{Cuadro $\mathrm{N}^{\circ} 4$}

AMGS: inmigrantes y emigrantes según tipos de origen y destino, respectivamente, migrantes intrametropolitanos y no migrantes, 1977-1982, 1987-1992, 1997-2002 y 2002-2006, población de 5 años y más

\begin{tabular}{|c|c|c|c|c|c|}
\hline Tipo de corriente migratoria & $\begin{array}{l}1977 \\
1982\end{array}$ & $\begin{array}{l}1987 \\
1992\end{array}$ & $\begin{array}{l}1997 \\
2002\end{array}$ & $\begin{array}{l}2002 \\
2006\end{array}$ & $\begin{array}{l}2004 \\
2009\end{array}$ \\
\hline Inmigración desde comunas no RM & 232.119 & 210.837 & 201.289 & 106.235 & 119.284 \\
\hline Inmigración comunas RM fuera del AMGS & 17.944 & 22.443 & 26.359 & 20.635 & 12.827 \\
\hline Inmigración al AMGS & 250.063 & 233.280 & 227.648 & 126.870 & 132.111 \\
\hline Emigración comunas no RM & 114.218 & 161.514 & 218.771 & 122.997 & 38.383 \\
\hline Emigración comunas RM fuera del AMGS & 20.912 & 29.248 & 58.251 & 33.206 & 29.731 \\
\hline Emigración del AMGS & 135.130 & 190.762 & 277.022 & 156.203 & 68.114 \\
\hline Migración intrametropolitana & 473.596 & 795.589 & 779.642 & 463.633 & 444.384 \\
\hline No migrantes (“inmóviles" AMGS) & 2.775 .078 & 3.023 .783 & 3.784 .381 & 4.250 .294 & 4.956 .759 \\
\hline Saldo migratorio & 114.933 & 42.518 & -49.374 & -29.333 & 63.997 \\
\hline Saldo migratorio "cercano" & -2.968 & -6.805 & -31.892 & -12.571 & -16.904 \\
\hline Saldo migratorio "Iejano" & 117.901 & 49.323 & -17.482 & -16.762 & 80.901 \\
\hline
\end{tabular}

Fuente: Rodríguez (2008) y procesamiento especial de la encuesta CASEN 2009.

La reconfiguración del AMGS: la migración como motor de la expansión periférica pero también de la recuperación del atractivo del centro y del cono oriente

La CASEN 2009 ratifica la importancia cuantitativa de la migración intrametropolitana, que supera largamente el intercambio del AMGS con el resto del país (incluso sumando entorno cercano y lejano). Aunque obviamente esta migración tiene un efecto nulo sobre el crecimiento de la ciudad -la gente se mueve en su interior- sí puede tener $-y$ de hecho ha tenido- efectos poderosos sobre la expansión territorial de la ciudad, despoblando zonas, densificando otras y empujando los límites de la ciudades en la comunas periféricas. El análisis de esta migración, como siempre, resulta crucial para entender la dinámica de crecimiento territorial desagregado de la ciudad.

En tal sentido, los resultados en materia de migración intrametropolitana de la CASEN 2009 permiten concluir que, salvo algunas excepciones, la estructura migratoria de las comunas del AMGS se mantiene similar a la descrita con base en la CASEN 2006 (Rodríguez, 2008, mapa 1). El cambio más importante es el paso de la comuna de Santiago de su histórica condición de expulsión a una de inmigración neta, y con montos no menores, como se verá más adelante (Figura $N^{\circ} 1$ ).

La Florida y Peñalolén también son comunas que cambian de condición, ya que la CASEN 2009 las muestra ahora con inmigración neta, lo que se asocia a renovación inmobiliaria dirigida a distintos sectores sociales, por lo que podrían consolidarse como comunas mixtas. Extrañamente, Macul se mantiene con emigración neta, pese a su reciente auge de construcción de edificios; para los años que siguen hasta el censo de 2012, los efectos del terremoto del 27 de febrero en materia de preferencias residenciales en torres y edificios de altura pueden agudizar esta emigración neta. Otras comunas también recuperan atractivo (La Pintana, San Ramón, Cerrillos), pero la falta (o desconocimiento) de factores estilizados (con excepción de la llegada del Metro a algunas de ellas) que explique esta transformación genera dudas sobre las perspectivas estratégicas de este cambio. 
Respecto de las comunas expulsoras, en general se mantienen ampliamente concentradas en el pericentro, el que salvo por el conducto centro-oriente sigue perdiendo población por migración, y esto pese a la extensión del subsidio para renovación urbana hacia muchas de estas comunas. El único caso Ila- mativo de comuna que deviene de emigración neta es San Bernardo, doblemente Ilamativo por ser periférica y además con importantes reservas de suelo urbanizable. Se perfila como estudio de caso para futuras investigaciones.

Figura $\mathrm{N}^{\circ} 1$

Comunas del AMGS (34), según condición de atracción o expulsión de migrantes, 1997-2002, 2002-2006, 2004-2009

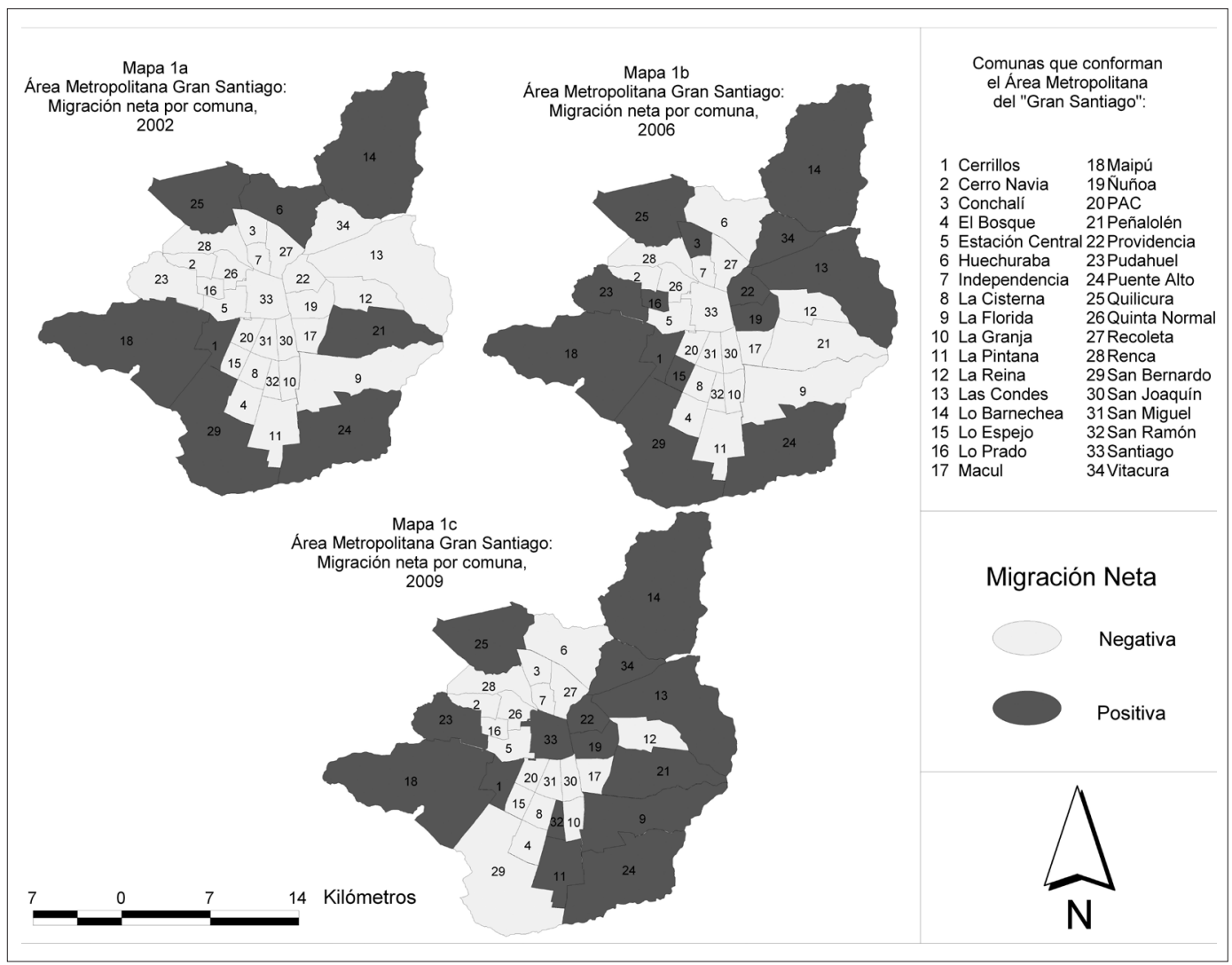

Fuente: Rodríguez (2008: 86: mapas 1a y 1b); mapa 1c elaborado por Daniel Espinoza con base en procesamientos especiales de la base de datos de la CASEN 2009.

Nota: Los mapas no corresponden a las dimensiones específicas de cada comuna pues algunas han sido recortadas para facilitar su despliegue.

No obstante la continuidad general, las modificaciones que muestra la CASEN 2009 obligan a una ligera revisión de la tipología presentada en 2008. En el Cuadro $N^{\circ} 5$ se expone esta versión revisada de la tipología, que deberá ser revisada nuevamente con la información que provea el censo de 2012.
11 Dado que la condición atractiva o expulsora deriva del saldo migratorio total de la comuna, el aumento de las comunas pericentrales "atractivas" puede deberse a la recuperación general del atractivo del Gran Santiago. El censo 2012 dará más pistas sobre las proyecciones de este nuevo estatus. 


\section{Cuadro $\mathrm{N}^{\circ} 5$}

Tipología ad hoc de comunas del Gran Santiago según localización, crecimiento y condición socioeconómica

\begin{tabular}{|l|l|}
\hline Centro & Santiago \\
\hline Pericentro expulsor & $\begin{array}{l}\text { Cerro Navia, Conchalí, Estación Central, Independencia, La Cisterna, La } \\
\text { Granja, Lo Espejo, Lo Prado, PAC, Quinta Normal, Recoleta, Renca, San } \\
\text { Joaquín, San Miguel, San Ramón, Macul. } \\
\text { Las Condes, Lo Barnechea, Ñuñoa, Providencia, Vitacura, La Reina. } \\
\text { Cono Oriente }\end{array}$ \\
\hline Periferia expansivá, Pudahuel, Quilicura, Puente Alto, La Pintana. \\
\hline $\begin{array}{l}\text { Periferia no } \\
\text { atractiva }\end{array}$ & \begin{tabular}{l} 
El Bosque, Huechuraba, San Bernardo. \\
\hline $\begin{array}{l}\text { Pericentro no } \\
\text { expulsor }\end{array}$
\end{tabular} \\
\hline
\end{tabular}

Fuente: Elaboración propia.

El procesamiento de los microdatos de la CASEN 2009 permite estudiar desagregadamente, con ciertas precauciones muestrales, el comportamiento migratorio de cada una de las comunas. En parte para evitar riesgos muestrales, pero también para articular el análisis en torno a la agrupación de comunas expuestas en el Cuadro $N^{\circ} 6$-que tiene mayor densidad teórica y mayor alcance de política que el examen individual de cada por comuna y que además permite exámenes más abreviados y estilizados-, se agruparon las comunas del centro, del cono oriente, de la periferia expansiva, de la Región Metropolitana que están fuera del AMGS y de las otras regiones del país; las comunas del resto del AMGS (el pericentro expulsor y no expulsor y la periferia no atractiva) se reunieron en un grupo ad hoc. Los resultados se exponen en el Cuadro $N^{\circ}$ 6, que corresponde a una matriz de migración entre todos estos componentes. 


\section{Cuadro $\mathrm{N}^{\circ} 6$}

Matriz de migración del AMGS según clasificación ad hoc de las comunas del AMGS y del resto del país

\begin{tabular}{|c|c|c|c|c|c|c|c|c|}
\hline \multirow{2}{*}{$\begin{array}{l}\text { Residencia en } \\
\text { noviembre de } \\
2009\end{array}$} & \multicolumn{8}{|c|}{ Residencia en Noviembre de 2004} \\
\hline & Santiago & $\begin{array}{c}\text { Cono } \\
\text { Oriente }\end{array}$ & $\begin{array}{l}\text { Periferia } \\
\text { Expansiva }\end{array}$ & $\begin{array}{l}\text { Resto del } \\
\text { AMGS }\end{array}$ & $\begin{array}{l}\text { Resto Región } \\
\text { Metropolitana }\end{array}$ & $\begin{array}{l}\text { Comunas } \\
\text { Otras } \\
\text { Regiones }\end{array}$ & $\begin{array}{l}\text { Total en } \\
\text { Noviembre } \\
\text { de } 2009 \mid\end{array}$ & $\begin{array}{c}\text { Migración } \\
\text { Neta }\end{array}$ \\
\hline Santiago & 113.748 & 7.613 & 1.351 & 8.679 & 987 & 12.670 & 145.048 & 19.440 \\
\hline Cono Oriente & 1.256 & 691.565 & 5.823 & 22.783 & 4.092 & 43.553 & 769.072 & 23.198 \\
\hline $\begin{array}{l}\text { Periferia } \\
\text { Expansiva }\end{array}$ & 646 & 5.024 & 1.821 .277 & 125.798 & 4.075 & 20.246 & 1.977 .066 & 88.726 \\
\hline $\begin{array}{l}\text { Resto del } \\
\text { AMGS }\end{array}$ & 3.364 & 37.757 & 40.089 & 2.514 .370 & 3.673 & 42.815 & 2.642 .068 & -67.367 \\
\hline $\begin{array}{l}\text { Resto Región } \\
\text { Metropolitana }\end{array}$ & 1.366 & 626 & 9.083 & 18.656 & 706.760 & 10.285 & 746.776 & 17.709 \\
\hline $\begin{array}{l}\text { Comunas } \\
\text { Otras } \\
\text { Regiones }\end{array}$ & 5.228 & 3.289 & 10.717 & 19.149 & 9.480 & 9.333 .042 & 9.380 .905 & -81.706 \\
\hline $\begin{array}{l}\text { Total en } \\
\text { Noviembre de } \\
2004\end{array}$ & 125.608 & 745.874 & 1.888 .340 & 2.709 .435 & 729.067 & 9.462 .611 & 15.660 .935 & - \\
\hline
\end{tabular}

Fuente: procesamientos especiales de la base de datos de la CASEN 2009.

\section{Periferia expansiva}

Es el sector que, por lejos, lidera el atractivo migratorio dentro del AMGS. Ahora bien, la magnitud de su migración neta se debe principalmente a migraciones intrametropolitanas, en específico, desde el pericentro expulsor y la periferia no expansiva (resto del AMGS en el cuadro $N^{\circ} 6$ ). De hecho, estos sectores le aportan un saldo migratorio mucho mayor que el balance que registra con las comunas de otras regiones. Los números son contundentes: el saldo migratorio entre el resto del AMGS y la periferia expansiva asciende a 85.709 personas, favorable a esta última, mientras que el balance con el resto de las regiones suma solo 9.529. Se ratifica plenamente, entonces, que el avance del poblamiento periférico de Santiago obedece principalmente a fuerzas centrífugas de la ciudad, toda vez que sigue estando basado en un desplazamiento masivo de población desde pericentro expulsor hacia la periferia, y no a la llegada de nuevos residentes desde el resto del país, como ocurría hasta la década de 1970 (Alberts, 1977).

Ahora bien, esta imagen de empuje interno del avance del poblamiento periférico debe ser matizada ligeramente a la luz de las cifras de la CASEN 2009, pues la periferia expansiva no tiene un atractivo unánime. En efecto, frente a la comuna de Santiago, al cono oriente y a las comunas fuera de la Región Metropolitana, pierde gente. De esta forma, la evidencia recogida por la CASEN 2009 ratifica la secular expansión periférica pero tiende a descartar cualquier protagonismo cuantitativo de la suburbanización de las familias de altos ingresos en este proceso. Además la CASEN 2009 muestra novedades respecto de la emigración de la comuna central hacia la periferia expansiva que pueden estar vinculadas a políticas urbanas implementadas 
desde hace varios años, las de renovación y repoblamiento de zonas céntricas, y cuyos efectos en materia de atractivo migratorio habían tardado en materializarse.

\section{Cono oriente}

Este sector está conformado por las comunas con la composición socioeconómica más acomodada de la ciudad y en él reside virtualmente la totalidad del grupo social más poderoso y pudiente del país (la alta burguesía en el sentido clásico del término) y la mayor parte de los hogares del decil superior de ingreso ${ }^{12}$ (estrato socioeconómico superior). Se constituye como un nicho de residencia para la clase alta, concentrando la mayor cantidad de personas de altos ingresos, reuniendo la mayor cantidad del empleo de la ciudad, con una alta cotización del suelo, ofertas de servicios privados de alto costo (clínicas, colegios, hoteles, etc.), entre otros. Además goza de un prestigio o estatus simbólico adquirido a través de los años, que no es traspasable a otras comunas, aunque estas experimenten rápidos procesos de ascenso socioeconómico.

12 Exactamente el 61,4\% de los hogares del decil superior de ingresos (jerarquía regional) reside en estas 6 comunas, que sumadas contienen al 15,8\% de los hogares del AMGS (procesamiento especial, CASEN 2009). Al respecto, el mismo indicador según la CASEN 2006 era de $56 \%$ en el caso de los hogares del decil $\mathrm{X}$ y de $15,5 \%$ en el caso del total de hogares. Abiertamente, estas cifras chocan con los planteamientos (y la evidencia en que se apoyan) relativos a la "dispersión territorial de la elite", que frecuentemente se repite en la bibliografía reciente sobre Santiago: "...la difusión espacial de enclaves de estatus socioeconómicos alto por áreas vecinas de estatus socioeconómico más bajo está plenamente constatada, al menos en sectores de la ciudad que ofertan condiciones ambientales atractivas al emplazamiento de estos grupos socioeconómicos" (Escolano y Ortiz, 2007: 67); "La construcción de "condominios cerrados" para grupos medios y altos fuera de su área tradicional de concentración, es tal vez el cambio más notorio y generalizado que está afectando a la estructura interna de las ciudades chilenas y latinoamericanas" (Sabatini et al., 2001). Evidentemente, no se trata de negar los procesos descritos en ambas citas, pues son reales. El punto es que de acuerdo a la evidencia disponible, este desplazamiento de familias de altos ingresos hacia ciertas zonas de la periferia y de los suburbios del AMGS, no ha revertido la histórica concentración de la elite en el cono oriente.
Una de las sorpresas que trajo consigo la CASEN 2006 fue la constatación de que todas las comunas del cono oriente, salvo La Reina, tuvieron saldo migratorio positivo entre abril de 2002 y noviembre de 2006. En el análisis de esas cifras se dejó constancia que se trataba de algo más que una sorpresa. En efecto, se trata de un hecho que se aparta de las predicciones teóricas y que parece ir en contra de factores estructurales (económicos, políticos y geomorfológicos, tal como se describen en Rodríguez, 2008: 88-89) que debieran desincentivar la llegada de nuevos habitantes. Incluso más, el hallazgo de 2006 -amén de novedoso porque el censo de 2002 mostraba un panorama muy diferentes, ya que todas las comunas del cono, salvo Lo Barnechea, registraban emigración neta- era contrario a la visión predominante entre los investigadores (no siempre bien respaldada en evidencia representativa, eso sí) de que el cono oriente tenía una hemorragia cuantiosa e inexorable dada por la suburbanización de familias de clase alta hacia Huechuraba, Peñalolén, Chicureo y otras zonas de la periferia y de los suburbios del área metropolitana (Sabatini y Cáceres, 2004). Los datos de la CASEN 2009, examinados ahora como cono oriente agregado, sugieren que el carácter atractivo de esta zona se ha consolidado, pues registra un saldo migratorio positivo superior a 20 mil y todas las comunas que lo integran, nuevamente con la excepción de La Reina, registran inmigración neta.

Ahora bien, el Cuadro $N^{\circ} 6$ revela un rasgo muy importante del atractivo del cono oriente: descansa en el magnetismo que ejerce para población que reside fuera del AMGS (esto es, resto de la RM y resto del país). Tanto es así, que en el intercambio con la comuna de Santiago así como en el intercambio con el "resto del AMGS", el cono oriente registra emigración neta. Tiene una ganancia ínfima con la periferia expansiva (la que, por el signo, no deja de ser llamativa, en todo caso). Pero alcanza un monto positivo firme en el intercambio con el resto de la Región Metropolitana, el que se transforma en una ganancia cuantiosa en el caso del intercambio con otras regiones, por cuanto su saldo llega a 40.264 .

Por estos resultados el atractivo del cono oriente adquiere otras connotaciones, que 
superan los antecedentes expuestos en la Introducción de este documento. Si bien está claro que la década de 2000 demostró que muchas de las limitaciones objetivas para la expansión y atracción de esta zona fueron superadas por una pujanza inmobiliaria -tanto vertical como horizontal en los ámbitos precordilleranos o de piedemonte directamente-, la evidencia que proporciona la CASEN 2009 sugiere que la demanda por residir en el cono oriente no se limitaba a la capital, sino que se extendía a otras regiones del país.

Este hallazgo obliga, entonces, a dos tipos de indagaciones que rebasan los alcances de este artículo, pero que serán abordados en futuras investigaciones. El primero es el análisis de los inmigrantes provenientes desde otras regiones. Si bien su figuración no es novedad, ya que desde el censo de 1982 se aprecia una migración neta positiva del Cono Oriente con el resto de las regiones del país (Figura $\mathrm{N}^{\circ} 1$ ), no hay análisis recientes de este flujo. En particular, su estatus habitacional (propietario/ arrendatario) y su actividad (estudiante, activo, jubilado) podría ser indicativo del tipo de vinculación que establecen con la ciudad ${ }^{13}$. Su nivel socioeconómico, en todo caso, ha de ser elevado, pues definitivamente hay que tener ingresos altos para residir en la zona de la ciudad con las propiedades y alquileres más elevados y que por su marcada homogeneidad social, virtualmente carece de espacios o zonas de bajo costo. Por cierto, lo anterior no aplica en el caso de las empleadas domésticas, en particular las que han migrado para trabajar "puertas adentro"; sin embargo, los

13 Análisis preliminares al respecto muestran un marcado sesgo de estos migrantes a ser arrendatarios, lo que puede ser indicativo de una estadía transitoria o de un proceso de acumulación tendiente a la adquisición posterior de una residencia propia. Por otra parte, también presentan un sesgo hacia la inactividad, lo que puede deberse a una mayor proporción de estudiantes. Con todo, dentro del cono oriente el perfil del inmigrantes desde otras regiones del país varían bastante entre comunas, teniendo Vitacura un perfil mucho más "enraizado" (migrantes propietarios, en rigor que residen en hogares que declaran ser propietarios de la vivienda, y económicamente activos) que Las Condes, por ejemplo (pero no cabe sacar conclusiones apresuradas al respecto por la escasa representatividad de la muestra en estos cruces complejos). El censo de 2012 proporcionará evidencia más firme sobre algunos de estos rasgos. mismos datos de la CASEN 2009 muestran que esta migración $\mathrm{NO}$ es decisiva ya que solo 2.900 de los 40.553 inmigrantes de otras regiones al cono oriente trabajaban en el servicio doméstico ${ }^{14}$.

Y el segundo es el de los migrantes intrametropolitanos, no tanto por su volumen o por su saldo -que, como se desprende del Cuadro $\mathrm{N}^{\circ} 6$, no se trasladan masivamente al cono oriente $y$, de hecho, este último tiene emigración neta en su intercambio con la comuna de Santiago y con el pericentro expulsor + periferia no expansiva- sino por sus características, ya que los costo de arrendar o comprar una vivienda allí introducen una alta selectividad socioeconómica a este flujo, y eventualmente otras selectividades que pudieran explicar porque se opta por el cono oriente y no por las urbanizaciones de lujo y condominios cerrados fuera de esta zona.

\section{Pericentro expulsor y periferia no atractiva}

Estos sectores conforman la categoría "Resto del AMGS" en el Cuadro No 5, por cuanto en la clasificación de Rodríguez (2008) los unifica su carácter expulsor, el que se mantiene según la CASEN 2009. Como ya se indicó al analizar la periferia expansiva, esta condición se debe principalmente a la ingente pérdida de población que experimentan estos ámbitos en su intercambio con la periferia. De hecho, el traslado hacia la periferia expansiva es masivo, pues casi 126 mil personas efectuaron tal mudanza en el período 2004-2009; como la contracorriente superó ligeramente las 40 mil personas, este puro intercambio implicó la pérdida de 86 mil persona para esta zona de la ciudad.

Como contrapartida, esta zona resulta atractiva -al menos en el período 2004-2009,

14 Para estimar la población que trabaja en servicios doméstico se usó la variable c_o13 (Código CIIU de actividad principal a 4 dígitos) de la base de datos CASEN 2009, cuya cifras no coinciden con las otras dos variables que podrían ser usadas con el mismo propósito C_o12 (Código CIUO de ocupación u oficio principal a 4 dígitos) y O23 ("En su ocupación principal, ¿usted trabaja como?). Los resultados relevantes para este estudio, en todo caso, no cambian si se usa alguna de esas otras dos variables. 
que tuvo como rasgo saliente la inmigración neta del AMGS- para las personas residentes en otras regiones. En efecto, si bien algo más de 19 mil personas se dirigieron desde estas zonas a otras regiones del país, la corriente principal fue en el sentido inverso y aportó más de 40 mil nuevos residente a estas zonas.

Tal vez lo más llamativo sea el saldo migratorio positivo que estas zonas registraron con el cono oriente. Un análisis preliminar desagregando las comunas de esta zona ${ }^{15}$ muestra que estos resultados se basan en el efecto de ciertas comunas en proceso de "ascenso o renovación sociorresidencial" -en particular Huechuraba, Peñalolén y La Florida y en menor medida San Miguel-, que reciben corrientes no menores desde el cono oriente. Nuevamente este flujo es un asunto para ser estudiado en investigaciones ulteriores $\mathrm{O}$, al menos, tenerlo presente para análisis específicos cuando se disponga del censo de 2012.

\section{Comuna de Santiago}

La comuna de Santiago presenta, por primera vez en muchas décadas, una migración neta positiva. La relevancia y la novedad de este dato, amerita una revisión más exhaustiva, que se hace, preliminarmente, a continuación. Cabe destacar que estudios anteriores ya habían Ilamado la atención sobre la incongruencia entre las cifras migratorias de la comuna y el dinamismo inmobiliario que presenta desde la década de 1990 (Rodríguez, 2008). Asimismo, análisis especializados de los patrones migratorios basados en datos del censo y de la encuesta CASEN 2006 mostraban indicios de una clara atenuación del carácter expulsivo de esta comuna (Rodríguez, 2007).

En tal sentido, la CASEN 2009 confirma estos estudios y análisis previos y sitúa a la comuna de Santiago con un saldo migratorio positivo elevado, una tasa de inmigración neta sobresalientemente alta y una relación de ganancia migratoria con todos los otros ámbitos identificados en el Cuadro $N^{\circ} 5$.

\footnotetext{
15 Datos disponibles, aunque no mostrados en este artículo.
}

Tal vez la cifra más sorprendente del Cuadro $\mathrm{N}^{\circ} 5$ respecto de la comuna de Santiago es el saldo positivo que registra con "las comunas de otras regiones", toda vez que en los tres censos previos, así como en la CASEN 2006, este balance había sido muy negativo, en gran medida por una sobreestimación originada en la homonimia de la ciudad/ localidad y la comuna. Como ahora esto no acontece, solo cabe preguntarse si en la capacitación de los encuestadores el problema de la homonimia fue tratado y se resolvió por esa vía. Explicaciones alternativas no son sencillas de hallar.

\section{Discusión final y conclusiones}

Tratando de poner en la balanza los factores relativos de atracción y expulsión de población de las 15 regiones de Chile, la evidencia que proporciona la CASEN 2009 vuelve a introducir dudas sobre los potenciales efectos desconcentradores de población del modelo de desarrollo imperante en el país desde hace más de tres décadas y que descansa fuertemente en la explotación de materias primas y bienes primarios. En esta ocasión, por vez primera se pueden apreciar los efectos migratorios de la crisis de una de las industrias boyantes del modelo imperante, a saber la del salmón, que se tradujo en una clara pérdida de atractivo de la región en que se asienta el grueso de dicha industria (X Región de Puerto Montt). Por otra parte, se ratifica que incluso períodos largos de precios altos de los minerales que exporta Chile (cobre, molibdeno, oro, plata, litio, entre otros) y que se traducen e ingentes ingresos para las grandes empresas del sector y sus trabajadores no se traducen en desplazamiento masivos hacia las regiones donde se explotan los minerales. Además de un hábitat que no es precisamente hospitalario y que en general limita la migración familiar, otras particularidades que erosionan el atractivo de su dinamismo económico son: a) se trata de actividades altamente intensivas en capital y en menor medida de trabajo (aunque con encadenamientos no menores); b) parte importante de su demanda de trabajo es temporal o puede ser satisfecha mediante sistema de turnos o estadías, lo que inhibe la migración propiamente tal; c) por lo anterior y por la estructura empresarial de los grandes actores del negocio, una fracción 
significativa de las ganancias no quedan en las regiones donde se asientan las actividades productivas.

Por su parte, en el período de referencia la RM y el AMGS avanzaron en el control de algunos de los factores de expulsión. El desempleo se mantuvo bajo la media regional, los índices de contaminación siguieron bajando, la nueva política habitacional logró mantener crecientes cantidades de beneficiarios de subsidio en sus comunas de origen, el plan de renovación urbana de la comuna de Santiago mantuvo protagonismo, y diversas obras de mejoramiento y vialidad urbanas lograron reactivar barrios, ampliar los espacios públicos y mejorar la conectividad. Tal vez el único ámbito en el que la población de la ciudad experimentó adversidades emergentes fue en el transporte público, por las secuelas, sobre todo iniciales, generadas por la implementación del Transantiago; con todo, no hay signos de efectos emigratorios (al menos de emigración hacia fuera de la región) de este plan de transporte colectivo.

Como interpretación inicial, esta combinación de factores que operan tanto en las regiones como en Santiago parecen clave para entender la evolución de los flujos migratorios entre regiones que muestra la CASEN 2009.

Por otro lado, con relación a la configuración territorial del AMGS, el motor migratorio del crecimiento demográfico de la periferia expansiva del AMGS sigue siendo el pericentro, que predominantemente alberga a sectores populares y medios. Más aún, en su intercambio con el sector oriente de la ciudad -tradicional asentamiento de los grupos acomodados- la periferia expansiva pierde población. Se trata de datos que relativizan la visibilidad de los desplazamientos de familias de la elite hacia zonas de la periferia bajo modalidades residenciales de condominios cerrados o parcelas de agrado, pues estos no alcanzan aún un peso cuantitativo capaz de modificar el carácter predominantemente pobre de la periferia de Santiago. Sin duda lo han modificado y lo han hecho más variopinto, pero el predominio del sector oriente como locus del segmento más pudiente del país no parece correr riesgo.
Ahora bien, el crecimiento de Santiago ya no puede leerse solo en clave de expansión periférica y suburbanización.

Por una parte, está la emergencia de la comuna de Santiago como zona atractiva, con tasas elevadas y alcance transversal, pues con todos los otros ámbitos territoriales considerados en el análisis registra un saldo migratorio positivo. Será necesario hacer seguimiento de esta tendencia con nueva evidencia en el futuro, pero haber recuperado el atractivo residencial supone una cierta acumulación material y simbólica que genera expectativas de continuidad. En efecto, los servicios tienden a seguir a la gente y si la comuna de Santiago antes era poco confortable para los residentes, la llegada de nueva población debiera generar una renovación y reforzamiento de los servicios públicos y privados y con ello atraer a más inmigrantes. Otro tanto ocurre en materia simbólica, donde la idea de "vivir en el centro" pasa de ser rechazada a ser aceptada, atrayendo nuevos migrantes, eventualmente muy selectivos en materia de ciclo de vida y perfil educativoocupacional. Evidentemente, esta retroalimentación positiva no está escrita en piedra y puede, finalmente, ser más bien transitoria.

Por otra parte se encuentra el renovado atractivo migratorio y la expansión inmobiliaria y demográfica de algunas (aún muy pocas, eso sí) comunas pericentrales. El impacto de políticas explícitas -como la ampliación del alcance territorial del subsidio de renovación urbana- y los efectos colaterales de otras decisiones (como la ampliación de la red de metro) parecen ser clave en esta articulación compleja de procesos, como lo ilustra el caso de La Florida.

Asimismo, el atractivo del sector oriente refuerza la idea de que es posible crecer redensificando y no solo extendiendo la ciudad. Claro está que en este caso conviven ambas opciones porque mientras es evidente la intensificación de la ocupación como resultado de la acelerada construcción en altura en Providencia, Las Condes y Ñuñoa también es conocida la ampliación de la frontera de la ciudad hacia la cordillera en comunas como Lo Barnechea, Vitacura y Las Condes. 
Finalmente, la aparición de emigración neta en algunas comunas periféricas, revela potenciales cuellos de botella de la expansión horizontal incesante. En algunos casos, estos pueden provenir de la misma institucionalidad, en particular de los límites urbanos de las ciudades. En otros, los factores de saturación pueden ser precisamente el alejamiento excesivo respecto de los puestos de trabajo o la aún insuficiente red de transporte colectivo. Y en más de algún caso puede estar influyendo la congestión vehicular que comienzan a experimentar cada vez con mayor regularidad las familias que se trasladaron a la periferia justamente para huir de tal congestión.

\section{Referencias bibliográficas}

ANGEL, S.; PARENT, J.; CIVCO, D. \& BLEl, A. Making Room for a Planet of Cities. Washington D.C: Lincoln Institute, 2011. Disponible en Internet: http://www.lincolninst. edu/pubs/1880_Making-Room-for-a-Planet-ofCities-urban-expansion.

ALBERTS, J. Migración hacia áreas metropolitanas de América Latina: un estudio comparativo. Santiago de Chile: CELADE, Serie E, No 24, CEPAL, 1977.

AROCA, P. Desigualdades regionales en Chile. Foreign Affairs Latinoamérica, 2009, Vol. 9, No 1, p. 53-62.

BANCO MUNDIAL. Informe sobre el desarrollo mundial 2009: Una nueva geografía económica. Washington D.C.: Banco Mundial, 2008.

Banco Interamericano de Desarrollo (BID). Urban sustainability in Latin America and The Caribbean. Washington D.C.: BID, 2011.

DAHER, A. Competencia: regiones ganadores y perdedoras en Chile. EURE, 1994, Vol. XX, N 60, p. 63-84.

DE MATTOS, C. Santiago de Chile, globalización y expansión metropolitana: lo que existía sigue existiendo. EURE, 1999, Vol.25, $\mathrm{N}^{\circ} 76$, p. 29-56.
DE MATTOS, C. Metropolización y suburbanización. EURE, 2001, Vol. 27, № 80, p. 5-8.

DE MATTOS, C. Globalización y metamorfosis metropolitana en América Latina. De la ciudad a lo urbano generalizado. Revista de Geografía Norte Grande, 2010, № 47, p. 81-104.

ESCOLANO S. y ORTIZ, J. Patrones espaciales de movilidad de la población: Algunos efectos en la sociogeografía del Gran Santiago. En: DE MATTOS, C. e HIDALGO, R. (Editores). Movilidad espacial y reconfiguración metropolitana. Santiago de Chile: Instituto de Estudios Urbanos, Pontificia Universidad Católica de Chile, Colección EURE-LIBROSInstituto de Geografía, Pontificia Universidad Católica de Chile, Serie GEOlibros N ${ }^{\circ} 8$, 2007, p. $53-68$.

FUENTES, L. Ciudad competitiva, ¿ciudad cohesionada? Vinculación entre competitividad urbana y cohesión social en Santiago de Chile. En: De MATTOS, C. y LUDEÑA, W. Lima_Santiago (Editores). Reestructuración y cambio metropolitano. Santiago de Chile: Andros, 2011, p. 205-232.

FUJITA, M.; KRUGMAN, P. \& VENABLES, A. The Spatial Economy: Cities, Regions, and International Trade. Cambridge/Massachusetts: The MIT Press, 2000.

GALETOVIC, A. y JORDÁN, P. Santiago: ¿Dónde estamos? ¿Hacia dónde vamos? En: GALETOVIC, A. (Editor). Santiago dónde estamos y hacia dónde vamos. Santiago de Chile: Centro de Estudios Públicos (CEP), 2006, p. 25-69.

Instituto Latinoamericano y del Caribe de Planificación Económica y Social (ILPES). Economía y territorio en América Latina y el Caribe. Desigualdades y políticas. Santiago de Chile: CEPAL, LC/G.2385-P., 2009.

INGRAM, G. Patterns of metropolitan development: what have we learned? Washington D.C.: BIRF, Policy Research Working Paper, No 1841, 1997. 
MINISTERIO DE DESARROLLO SOCIAL. Encuesta CASEN. Santiago de Chile, 2010. Disponible en Internet: http://www.ministeriodesarrollosocial.gob.cl/casen/Estadisticas/ perfil_regional.html.

RODRÍGUEZ, J. Paradojas y contrapuntos de dinámica demográfica metropolitana: algunas respuestas basadas en la explotación intensiva de microdatos censales. En: DE MATTOS, C. e HIDALGO, R. (Editores). Movilidad espacial y reconfiguración metropolitana. Santiago de Chile: Instituto de Estudios Urbanos, Pontificia Universidad Católica de Chile, Colección EURE-LIBROS - Instituto de Geografía, Pontificia Universidad Católica de Chile, Serie GEOlibros No 8, 2007, p. 19-52.

RODRÍGUEZ, J. Dinámica sociodemográfica metropolitana y segregación residencial: ¿Qué aporta la CASEN 2006? Revista de Geografía Norte Grande, 2008, No 41, p. 81-102.

RODRÍGUEZ, J. y BUSSO, G. Migración interna y desarrollo en América Latina entre 1980 y 2005. Un estudio comparativo con perspectiva regional basado en siete países. Santiago de Chile: CEPAL. 2009.

SABATINI, F. y CÁCERES G. Los barrios cerrados y la ruptura del patrón tradicional de segregación en las ciudades latinoamericanas: El caso de Santiago de Chile. En: CÁCERES, G. y SABATINI, F. (Editores). Barrios Cerrados en Santiago de Chile: entre la exclusión y la integración residencial. Santiago de Chile: Lincoln Institute y Pontificia Universidad Católica de Chile, 2004, p. 9-43.
SABATINI, F.; CÁCERES, G. \& CERDA, J. Residential segregation pattern changes in main Chilean cities: scale shifts and increasing malignancy. Ponencia presentada en el International Seminar on Segregation in the City, Lincoln Institute of Land Policy, Cambridge, 25-28 de julio de 2001.

SASSEN, S. The Global City. Princeton: Princeton University Press, 1991.

SASSEN, S. El reposicionamiento de las ciudades y regiones urbanas en una economía global: empujando las opciones de políticas y gobernanza. EURE, 2007, Vol. 33, No 100 , p. 9-34.

TORRES, H. Social and environmental aspects of peri-urban growth in Latin American mega-cities. In: POPULATION DIVISION, DEPARTMENT OF ECONOMIC AND SOCIAL AFFAIRS, UNITED NATIONS SECRETARIAT. United Nations Expert Group Meeting on Population Distribution, Urbanization, Internal Migration and Development. Nueva York: ONU, ESA/P/WP.206, 2008. Disponible en Internet: http://www.un.org/esa/population/ meetings/EGM_PopDist/EGM_PopDist_Report.pdf. 
